Citation: Wang, X., Crookes, D., Harding, S, \& Johnston, D. (2020). Evaluating Audio Description and BPS Visitor Experience in Titanic Belfast. Journal of Audiovisual Translation, 3(1), 246-263.

Editor(s): A. Matamala \& J. Pedersen

Received: March 2, 2020

Accepted: October 12, 2020

Published: December 21, 2020

Copyright: (2020 Wang, Crookes, Harding \& Johnston. This is an open access article distributed under the terms of the Creative Commons Attribution License. This allows for unrestricted use, distribution, and reproduction in any medium, provided the original author and source are credited.

\section{Evaluating Audio Description and BPS Visitor Experience in Titanic Belfast}

\author{
(iD) Xi Wang ${ }^{\bowtie}$ \\ Queen's University Belfast, UK \\ Danny Crookes \\ Queen's University Belfast, UK \\ Sue-Ann Harding \\ Queen's University Belfast, UK \\ David Johnston ${ }^{\bowtie}$ \\ Queen's University Belfast, UK
}

\section{Abstract}

This paper presents the results of a study that evaluates audio description (AD) and visitor experience with a group of blind and partially sighted (BPS) visitors to a real-world visitor attraction-Titanic Belfast. We apply the 10-facet model of visitor experience of Packer and Ballantyne (2016) for the first time in the context of accessibility, and through this we highlight accessibility issues which arose during the study. We identify two categories in our qualitative analysis that the model (Packer \& Ballantyne, 2016) cannot cover. We also model the factors that influence visitor experience and apply them to the later approach of Packer, Ballantyne, \& Bond's (2018) Dimensions of Visitor Experience (DoVE) Adjective Checklist. The checklist is based on their previous 10-facet model, and translated and refined into 15 dimensions. Although the DoVE checklist is not specifically designed for the context of accessibility, we found that it is sufficiently comprehensive to model accessibility aspects of the museum $A D$ and visitor experience for BPS visitors.

Key words: audio description, accessibility, visitor experience, museums, BPS visitors.

\footnotetext{
Xi.Wang@qub.ac.uk,https://orcid.org/0000-0002-0588-1156

d.crookes@qub.ac.uk, https://orcid.org/0000-0002-3533-6095

\s.harding@qub.ac.uk, https://orcid.org/0000-0002-7662-7649

凶d.johnston@qub.ac.uk, https://orcid.org/0000-0003-3153-0162
} 


\section{Introduction}

Although there is already a great deal of scholarly writing on both film and theatre audio description (AD) and its reception (Fryer, 2016; Matamala \& Orero, 2016), there has been substantially less work carried out on $A D$ in museums, especially with a focus on the visitor experience of blind and partially sighted (BPS) visitors. Museum AD can still be described, in that sense, as an audiovisual translation (AVT) newcomer. In terms of human rights, however, $A D$ is crucial to meet the requirements and needs of BPS visitors in terms of quality access to museums, especially when AD is conceived not just as a verbal description for BPS visitors to access various visual content, but also as a means of enabling them to experience museums in a way that allows for similar levels of pleasure, learning and satisfaction to those experienced by the general population.

Our research aims to address this challenge by first seeking an understanding of the complex impact of museum $A D$ on visitor experience and, in particular, of emotional response. We did this by analysing the responses of 13 BPS visitors in a real museum setting-Titanic Belfast (located on the site where the Titanic was built, in Northern Ireland) - and evaluating the existing AD produced by a professional company. We determined the BPS visitors' emotional responses and assessed the quality of their visitor experience. We employed a mixed method of in-tour questions and an aftertour questionnaire for the evaluation of the AD. The qualitative data are analysed using the visitor experience models introduced by Packer and Ballantyne (2016).

This paper centres on the complexity of evaluating the AD and BPS visitor experience in a real scenario, and highlights generally transferable lessons in terms of designing more detailed AD for BPS visitors. The paper is structured as follows: firstly, we introduce the research context and basic concepts of accessibility quality and museum $A D$ in terms of visitor experience and emotional response; secondly, we introduce the methodology employed; and, finally, we discuss the results and their implications for future practice.

\subsection{Quality of Accessibility}

In legislation at national and international levels, accessibility is increasingly acknowledged as a human right. However, overall, the process of design for museum accessibility and its quality implementation remain slow (Greco, 2016).

Accessibility research must have as one of its central aims the assurance of a level of emotional experience and engagement that is comparable in quality to that of sighted visitors. For example, one of the BPS participants in our study observed other visitors responding to exhibits with emotions which she herself did not experience. After her first visit to Titanic Belfast, referring to the part of the exhibition that presents the sinking of the ship, she said: "Some of my family members were crying, but I was wondering why they cried". This comment speaks to the heart of quality. It is not 
just about access; it is access to the same quality of experience as enjoyed by those people for whom access legislation is not necessary.

We acknowledge that the experience of museums varies from person to person, and we recognise that different people bring diverse interests and perspectives to the experience (Falk, 2016); but we also note that BPS people are usually regarded as a homogenous group in terms of experience design. Consequently, we argue that the diversity of the conditions and degree of people's sight loss as well as their different cultural and social experiences must be taken into account in any design process.

Emotional engagement with experience (which, in turn, may derive from clarity of understanding relevant information and context) is one of our key concepts of quality access. This is also important for the audio describer, who has to present material in such a way that their audience is enabled to engage imaginatively with the $A D$. Consequently, audio describers have to look at what makes people's experience positive, how people engage with potentially emotional material, and how AD can ensure that BPS visitors have the same opportunities for engagement as the rest of the visitor population, and in ways that will speak to their imagination.

\subsection{Museum AD and Visitor Experience}

Museum AD is a tool used to facilitate and enhance accessibility to heritage centres and museums for BPS visitors. AD has been defined as follows:

$A D$ is a service for the blind and visually impaired that renders Visual Arts and Media accessible to this target group. In brief, it offers a verbal description of the relevant (visual) components of a work of art or media product, so that blind and visually impaired patrons can fully grasp its form and content (Remael, Reviers, \& Vercauteren, 2015, p. 9).

In other words, AD uses speech to make visual materials accessible to those who might not perceive the visual elements themselves. However, accessibility is not limited to visual artefacts in museums. Such venues are intended to create a site of meaningful, activist, discursive and intellectual exchanges between the widest possible ranges of people, and to enhance their physical, perceptual, cognitive and sensorial experience during the visit (Cachia, 2013). Museums, in this way, have begun to re-consider themselves as institutions concerned with sensorial experience rather than purely visual apprehension (Cachia, 2013).

Therefore, it is increasingly recognised that museum visitors, including BPS visitors, search for pleasure, entertainment and enjoyment as well as learning. In the specific case of the research context of this paper-Titanic Belfast-it is certainly true that a core aim of the exhibition is to provide a pleasant, entertaining and engaged experience for visitors. The venue is recognised internationally as a top tourist attraction, designated the World's Leading Tourist Attraction in 2016 (World's Leading Tourist Attraction, 2016), and is already committed to high accessibility standards, such as free-of-charge $A D$, hand rail extensions and contrasting floor textures (Access at Titanic 
Belfast, n.d.). In the year 2017/18, 841,563 visitors came through its doors ("A Titanic number of visitors," 2018).

Although Titanic Belfast has very strong museological qualities and a very strong museological commitment to exhibition, it is not just about information: it is an experience which is rooted in one of the great traumas of $20^{\text {th }}$ century history. It is especially poignant for Belfast and Northern Ireland, where the ship was designed, built and launched. Titanic Belfast explains itself as "the world's largest Titanic visitor experience, exploring the Titanic story in a fresh and insightful way" ("Explore," n.d.). With the support of digital technology, it highlights and features a structured and designed visitor experience, bringing visitors on board as though on to the ship, and guiding them through a series of galleries to discover and explore the whole story as it happened more than a century ago. One of the attractions is that Titanic Belfast is located on the very site where Titanic was built and launched; this adds to the potential for deep emotional engagement.

The visitor experience at Titanic Belfast is designed to be highly engaging, interactive and immersive. Titanic Belfast is thus somewhat different from a traditional museum, where artefacts are also stored and displayed (Alexander, Alexander, \& Decker, 2017). The six floors of the building feature nine interactive galleries: the first gallery-Boomtown Belfast-is on the first floor, and five galleries are on the fourth floor, including the Shipyard Gallery, the Launch Gallery, the Fit-Out Gallery, the Maiden Voyage Gallery, and the Sinking Gallery. Another three galleries are on the third floor: the Aftermath Gallery, the Myths \& Reality Gallery, and the Titanic Beneath Gallery. Because of the collectable and expensive nature of genuine Titanic artefacts, there are only a few actual artefacts on display. For example, the artefacts in the Maiden Voyage Gallery are: Dr Simpson's letter, the Hart family letters, and a first class menu. In three of the other galleries, including the Sinking Gallery, there are no artefacts. Instead, these nine galleries, "draw together special effects, dark rides, full-scale reconstructions and innovative interactive features" ("The Titanic Experience," n.d.).

The definition of museum $A D$ given at the beginning of this section seems too limited to define the quality of access in this specific context. As we have noted, museum $A D$ is not just a verbal description for BPS visitors to access the visual content, but it should also give equal access to the whole visitor experience in the museum. Hutchinson and Eardley (2019) conclude that the purpose of $A D$ should include enabling end-user access not only to things but also to the communicative and cognitive impact of the exhibited objects: in other words, to the engaged experience. This approach implies that the AD evaluation process should move beyond the focus on the AD itself, but must also evaluate the impact of the $A D$ on the whole experience of the visitor, including the emotional, participatory, interactive, and social experience.

Visitor experience, again as we have observed, can be different for each person. People come to museums bringing with them their past history, their own reasons for visiting, and their specific prior experience (Hooper-Greenhill, 2000). In their research, Falk and Dierking (2016) also point out that every visitor brings their own personal context, which includes their prior interests and beliefs, motivations and expectations, previous knowledge and experience, their own particular approach to 
choosing and controlling engagement, and to interacting with the social and physical context. Social context refers to accompanying visitors (e.g. friends or relatives), museum staff, and strangers. Physical context can be the physical space, a large scale environment in the museum, the architecture, the exhibitions, the content delivery and the events (Falk \& Dierking, 2000). These personal, social and physical contexts add to the complexity of understanding the visitor experience, especially given the factors of BPS visitors' different sight conditions. Thus, there is no single factor affecting the visitor experience, not even the $A D$, although the $A D$ tends to be one of the main resources along with tactile artefacts (such as carpets) and other audio-visual presentations. To better understand this complex visitor experience context, we opted to use a visitor experience model to demonstrate the multiple layers of visitor experience.

Packer and Ballantyne (2016) have produced a multifaceted model of visitor experience based on an analysis of visitor studies and broad leisure and tourism literature that attempts to characterise different types of visitor experience. Their model consists of ten facets of a visitor's experience including physical, sensory, cognitive, emotional, hedonic, restorative, introspective, transformative, spiritual and relational experience, illustrated below in Figure 1.

Figure 1.

Packer and Ballantyne's 10-Facet Model of the Visitor Experience

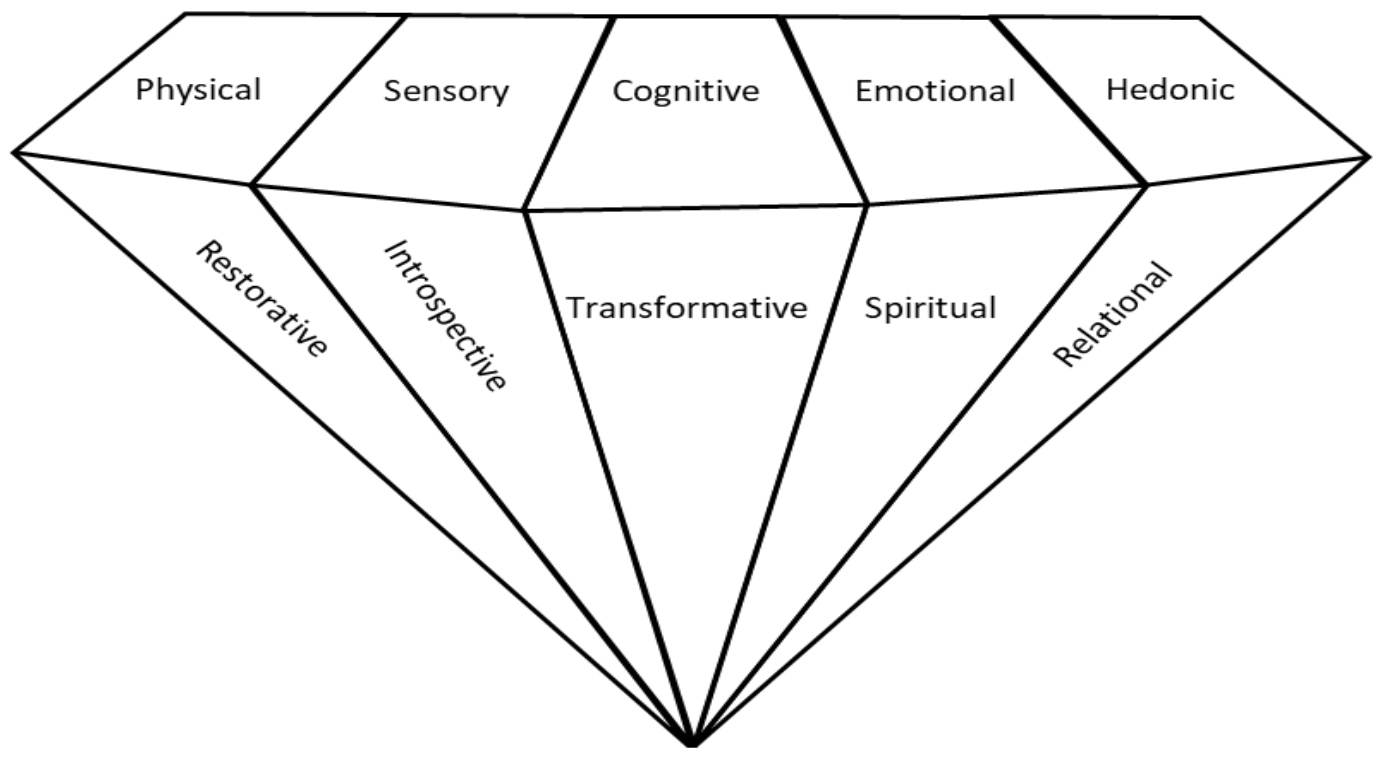

Source: Packer \& Ballantyne, 2016, p. 136.

This differs from other models, as cited in Packer and Ballantyne (2016), such as those that focus more on the takeaway outcomes (Aho, 2001), or on one specific experience, e.g. aesthetic experience (Csikszentmihalyi \& Robinson, 1990), pleasure (Dubé \& Le Bel, 2003), or memorable experiences (Kim \& Ritchie, 2014). In contradistinction, the Packer and Ballantyne model is more comprehensive when it comes to categorising the different experiences, and for this reason we propose its use, for the first time, for BPS visitors. As the authors point out: "just as cutting a gemstone allows its unique qualities 
to be revealed, considering the visitor experience in this way does not necessarily reduce it to components but allows the unique features of each facet to be observed and appreciated" (Packer \& Ballantyne, 2016, p. 135).

Packer and Ballantyne subsequently developed a DoVE (Dimensions of Visitor Experience) adjective checklist, in which the 10-facet model of visitor experience is translated into 15 dimensions. For each dimension, adjectives are listed in order to interpret and explain the dimension. This checklist was designed to quantitatively analyse visitor experience in various cultural venues and visitor attractions. In terms of the specific case of qualitative analysis of BPS visitors' experience addressed by this paper, both the 10-faceted model and the DoVE adjective checklist are used for evaluating the BPS visitors' experience, and a comparison between the two is discussed in Section 3.

\subsection{Museum AD and the Emotional Response}

Unlike previous research which focuses predominantly on the comprehension of end users, some current research addresses the impact of $A D$, particularly focusing on emotional responses and user experience. Such emotions, of course, are complex. Kleinginna (1981) defines them as follows:

Emotion is a complex set of interactions among subjective and objective factors, mediated by neural hormonal systems, which can (a) give rise to affective experiences such as feelings of arousal, pleasure/displeasure; (b) generate cognitive processes such as emotionally relevant perceptual effects, appraisals, labelling processes; (c) activate widespread physiological adjustments to the arousing conditions; and (d) lead to behaviour that is often, but not always, expressive, goal-directed and adaptive (p. 355; as cited in Matamala et al., 2020, p. 131).

In order to understand the role emotions can play within various museum contexts, research has been carried out in situ to investigate the impact of emotions on visitor satisfaction (Del Chiappa, Andreu, \& Gallarza, 2014), visitor experience (Alelis, Bobrowicz, \& Ang, 2013), and the free-choice learning process (Falk \& Dierking, 2000). Researchers point out that emotions play a determinant role in visitor satisfaction (Del Chiappa et al., 2014). Falk and Gillespie (2009) investigate the role of emotion in Science Centre visitor learning, demonstrating that "a particularly arousing experience ... not only can create elevated emotions amongst visitors but that this arousal might result in longterm positive changes in visitors' cognition, attitudes and behaviour" (p. 128). Bedigan (2016) looks at the relationship between emotions and visitor satisfaction, engagement, and education, and argues that emotions can play a significant role in learning and engagement if the experience can be more personalised; emotions can also play a significant role in memory formation regardless of whether they are experienced as positive or negative. These facts reinforce the importance of emotion in evaluating museum AD.

The methodology of recent user reception studies for evaluating the emotional response of BPS visitors to $A D$ has already gone beyond the sort of subjective perceptions usually elicited by questionnaires. Objective measures, such as using Galvanic Skin Response (GSR), heart rate (HR) or 
EEG, are employed; triangulation of the data is used both in audiovisual translation (AVT) and media accessibility (MA) research (Matamala et al., 2020). Such sensor-based approaches to automatic emotion detection have been shown to give effective results in a controlled environment; but they are much less effective in a real world environment where there can be multiple simultaneous emotions/mixed emotions being experienced (Berrios, 2019; Cruz-Garza et al., 2017).

Recent $A D$ reception research in film is starting to focus on the quality of experience of the $A D$ audience with an emphasis on emotion (Walczak \& Fryer, 2017). Emotional description can even be deemed as a new $A D$ style, differing from the traditional/standard AD which follows ethical guidelines that emphasise objectivity. Emotional description is a more narrative version, sometimes named audio narration (AN). Emotional AN is creative and subjective with the aim of enabling BPS audiences' access to the emotional experience offered by cinema to sighted viewers (Ramos, 2015). In her recent research, Ramos uses heart rate measurement to compare an objective AD with an emotive AN (Caro, 2016; Ramos, 2015). Her results show that not only is the BPS audience's emotional reaction much stronger while listening to emotive AN, but that audiences also accept and enjoy subjective AN (Ramos, 2015).

Given the findings above, the evaluation of visitors' personal emotional responses to the $A D$ and the surrounding environment can be part of evaluating the $A D$ and its impact on visitor experience. However, it is still the case that there is less attention paid in museum AD studies to emotional responses and visitor experience (Hutchinson \& Eardley, 2019; Neves, 2013).

There are several different approaches to the measurement of emotional states. According to Russell's classification model of emotion, emotional states are structured along dimensions, with arousal and valence as the horizontal and vertical axes (Russell, 1980). In the discrete emotions model, six basic human emotions are proposed: happiness, anger, fear, sadness, disgust, and surprise (Ekman, 1999; cited in Falk \& Gillespie, 2009). Note that this list of emotions is not exhaustive, and can depend on the domain. A third approach is to separate the positive and negative affect dimensions in order to understand the service satisfaction (Liljander \& Strandvik, 1997; cited in Del Chiappa et al., 2014).

\section{Methodology}

\subsection{Participants in the Titanic Belfast Study}

The participants were registered members of the Royal Institution of Blind People, Northern Ireland. A community worker with the RNIB acted as a gate keeper to recruit potential participants for this tour. In total, 13 BPS participants turned up and took part in the tour. The group represented different levels of sight loss, with 9 blind participants and 4 partially-sighted participants; 4 participants were 
born with sight loss and 9 participants had developed sight loss. Ages ranged from 26 to $75+$, and there was an equitable gender split (7 male and 6 female).

After planning the research in detail, an ethics application was submitted. The study was approved by the ethics committee of the School of Arts, English and Languages at Queen's University Belfast. All participants volunteered to take part in this study.

A structured tour of Titanic Belfast usually takes several hours. For the sake of time, we decided to focus on the fourth-floor part of the tour, where a whole story is narrated from the building of the Titanic to its sinking. The selected galleries, therefore, are the Shipyard Gallery, the Launch Gallery, the Fit-Out Gallery and the Sinking Gallery. The AD device was controlled by each participant using a small numerical keypad and the AD was delivered via individual headsets.

\subsection{Data Collection Strategies}

In this study, data collection was effected through a combined method including (i) open in-tour questions; and (ii) an after-tour questionnaire. The open in-tour questions-such as, how do you feel? - were asked by the tour guide after each gallery. Participants were free to express any of their ideas about their emotions, their visitor experience, their reception of the $A D$, and any particular points catching their attention, etc.

The questionnaire consisted of two sections: the first part asked for BPS participants' feedback on their visitor experience and on the AD. In this section, 15 questions were asked of the participants. Five were about the $A D$, including the narrator's voice, the helpfulness of the $A D$ in terms of participants' experiences, the amount of information in the $A D$, the language and terms used in the $A D$, and participants' suggestions as to how the $A D$ might be improved. Ten questions were asked targeting the participants' visiting experience, including the overall experience that participants had in different galleries, the ease of using the AD device, whether participants could hear clearly and understand the external audio commentary in the Ride and the Sinking Gallery, their haptic experience of the carpets and fabric books, their emotional experience in the Sinking Gallery, their overall experience of the tour, and any further comments they would like to make.

For the purposes of statistical analysis, the second section of this questionnaire asked factual questions relating to participants' age, gender, educational background, and levels of vision. Due to the various sight loss conditions of the participants, large print questionnaires were provided, and in the reception study, the consent form and questionnaire were read out to the participants (by participants' choice) and their feedback was noted on the questionnaire sheets by the person conducting the reception study (by participants' choice). The participants agreed to their comments and feedback being recorded during the reception study for subsequent checking and transcription. 


\section{Results and Discussion}

\subsection{Analysis of the Visitor Experience}

Once we had gathered and transcribed all the user feedback, we proceeded to apply the 10-facet Packer and Ballantyne model. Our method was to try to allocate each item of feedback to one of the 10 facets on the model shown in Figure 1 above (Section 1.2). We report below on the feedback on all 10 facets (although some facets had little feedback). In Figure 2, the facets are shown on the right, along with the number of feedback items allocated to each facet. We believe this to be the first research study that applies Packer and Ballantyne's multifaceted model of visitor experience in the context of accessibility for the purposes of analysing BPS visitors' experience (confirmed with Ballantyne and Packer, personal email communication, June 10, 2019).

Some of the feedback related not so much to the visitor experience, but to certain factors which influenced the visitor experience. We separated out these factors into three main categories (shown to the left of the yellow Visitor Experience box in Figure 2). Thus, Figure 2 shows two sides of the feedback on the BPS visitor experience: their perceived experience and factors which influenced their experience. There is, of course, some overlap between the two, so some feedback items are allocated to both sides. In the following sections, we give examples of feedback allocated, first to the factors (on the left), and then to the facets (on the right).

Figure 2.

Modelling the BPS Visitor Experience with Feedback Allocation

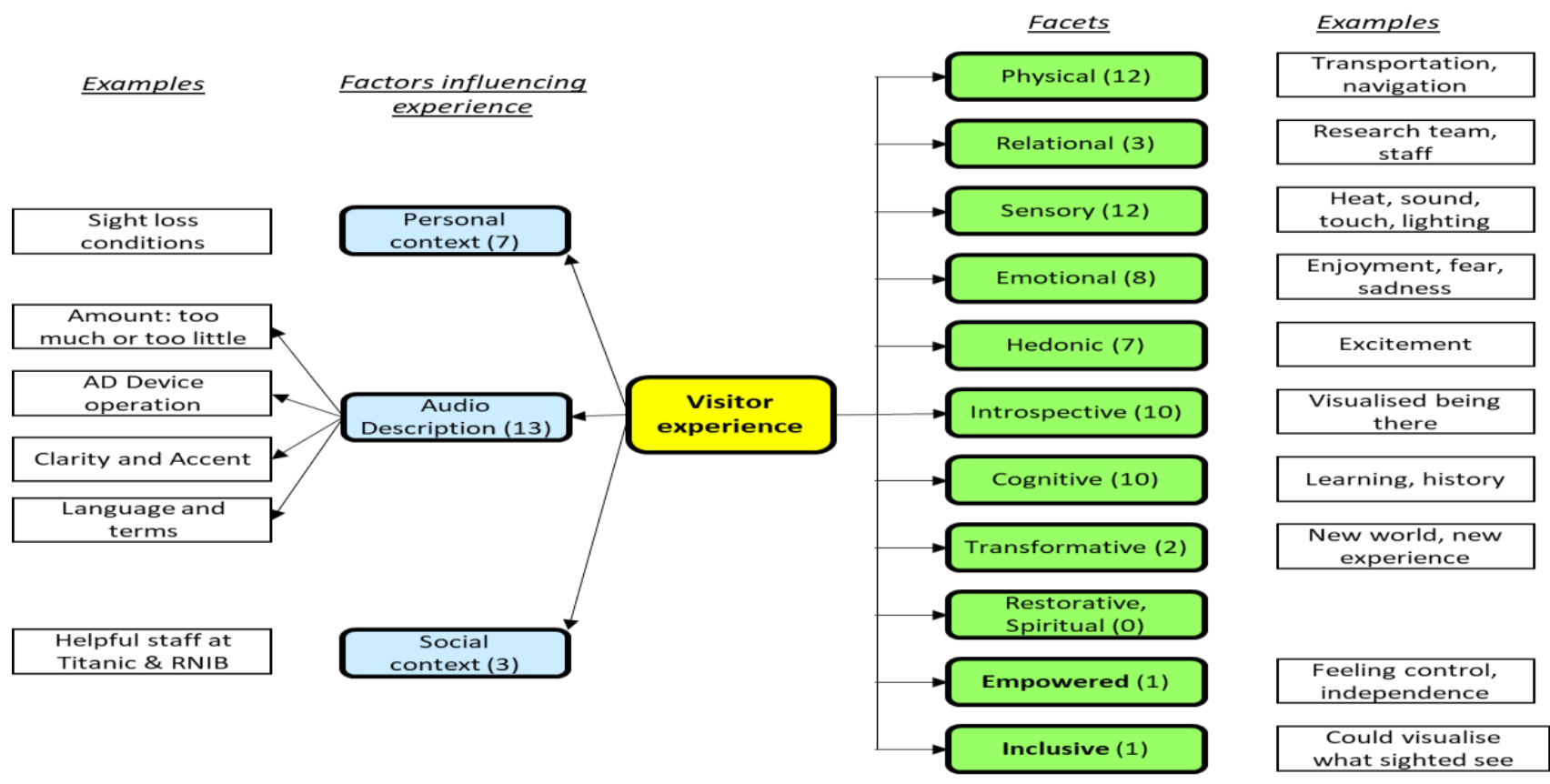




\section{Examples of Factors Which Have an Effect on Visitor Experience}

Falk and Dierking's interactive experience model (2016) is helpful for analysing the factors which impact on visitor experience. Apart from the factors previously mentioned in Section 1.2, this section focuses on factors commented on by the BPS visitors.

Firstly, the varying degrees of sight loss have a significant impact on BPS visitors' experience. For example, one of the partially-sighted people commented that it was unnecessary to describe colour because he could distinguish the colour, although blind participants found colour description helpful.

Secondly, a common theme in the feedback was the desire for additional $A D$ at certain points where it was perceived to be lacking. That said, participants had different opinions as to the desirable amount of $A D$. At several points, some thought there was too much $A D$, whereas others thought there was too little. For example, at the carpets in the Fit-Out Gallery, one person wanted more $A D$, whereas one said the tactile element spoke for itself. This reflects an ADLAB guideline: "prepare different DGs [descriptive guides] with different lengths, degrees of detail, layers of information to be used at will" (Remael et al., 2015). A solution would be for the amount of AD to be selected interactively by the user.

The feedback from participants on the AD was that it was generally simple and clear. The feedback basically covered three aspects: the detailed, the descriptive, and the simple and clear characteristics of the chosen AD. These key adjectives are consistent with the AD guidelines such as ADLAB (Remael et al., 2015) and Audio Description Guidelines and Best Practices (Snyder, 2010).

Thirdly, the keypad-based AD device was found to be unfamiliar to BPS visitors and to some extent to be difficult to use. Our analysis suggests that a natural language voice-driven AD device would be much more convenient in terms of usage.

Fourthly, the interaction with RNIB volunteers and staff at Titanic Belfast had an influence on BPS visitors' experience. In some cases, it is sometimes difficult to distinguish between the factors which influence the experience, and the experience itself. As mentioned, there was a significant overlap between the two. Thus, Section 3.1.3 provides additional feedback on visitors' relational experience.

\section{Examples of Physical Experience}

We found several examples related to the physical experience facet, including the transportation to the venue, finding the entrance to the venue, and internal and external navigation.

Participants said it was difficult to find the entrance to the venue after alighting from the bus. The need for navigational assistance outside the museum should not be overlooked-a factor which, of course, might be relevant to other museums. One visitor noted, in this regard, that "navigation information might be lost to a BPS person. It was difficult to find the entrance to the museum. Also, the water feature [a decorative feature near the venue entrance] might be a health and safety issue for BPS people". 
More internal navigation assistance, either through the AD or through other physical means (a rope or tactile floor) would also be valued by participants.

\section{Examples of Relational Experience}

The tour includes interaction with several people assisting with the visit. This did have some impact on the experience. For example, if one assistant was more helpful than others, they might give additional information which was not in the AD. Also, family members in some cases gave extra information to improve the experience of their relative, but this was not uniform.

\section{Examples of Multi-Sensory Experience}

Multi-sensory experience obviously dominates the visitor experience among BPS visitors. Most participants enjoyed the sound effects, heat, and tactile elements, in addition to the AD. All participants expressed the view that they particularly enjoyed the tactile elements. Touch is important, they noted, in order to learn and understand.

There were different, generally positive, views on the local accent of the external audio and the audio description. Most participants found the local accent enriched their experience, e.g. "I thought the local accent enhanced it"; "It was typical Belfast. You want that bit of culture"; "You can picture being in the shipyard. I can picture myself when I was 14 and was in the shipyard with my dad. The accents give you a sense of being in Belfast". One person recognised the voice of the professional narrator from other BPS commentaries, which made him feel more at ease.

\section{Examples of Emotional Experience}

Aspects of the feedback on the participants' emotional experience were more unexpected. In terms of the negative responses, three participants described their emotions as frightened, frustrated, or confused at their visit to the Ride. The Ride is a six-minute chair-lift type ride that travels downwards, twisting, turning, jerking and rotating through the noise, the heat, the lighting effects, the bustle, and video footage of shipyard workers (Irwin, 2012). A commentary by a local shipyard worker is heard throughout the journey, aiming to create an immersive experience for visitors to explore workers' real life at the shipyard (see Figure 3). The BPS participants stated that, as they could not see what was going on in the Ride, they were unsure as to what to expect. One participant after the Ride said, "Good, enjoyed it. Some parts confusing, some dark, the heat and the sound, I love the sound." Yet, during the reception study, he said "being visually impaired, I thought the sound effects good and the heat was very enjoyable" without referring to any confusion. However, another participant noted, "The heat and the noise made me afraid. It was awful". Interestingly, that same participant still rated the Ride as a positive experience in the questionnaire-a demonstration of the mix of simultaneous emotions. The in-tour questions reveal intermediate, short-term emotions, while the questionnaire reveals longer term emotions. 
Figure 3.

The Ride at Titanic Belfast

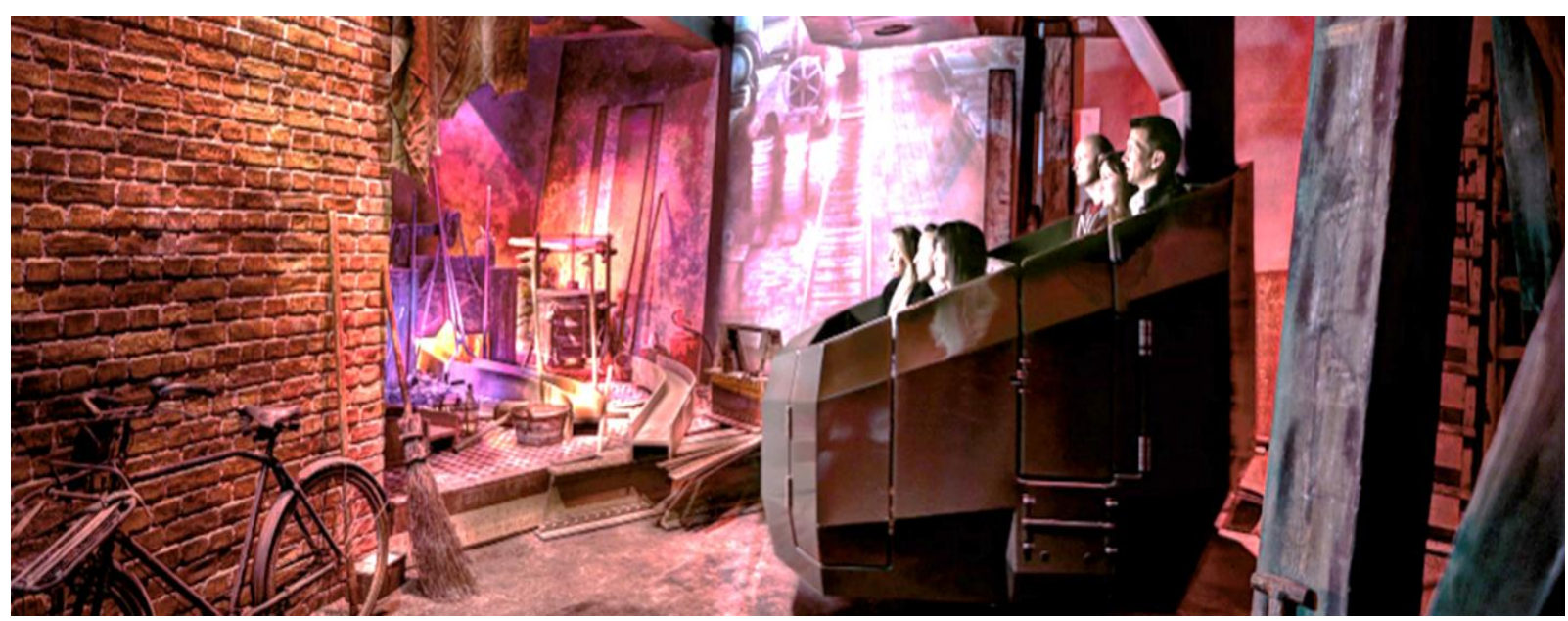

Source: "The Shipyard Ride," n.d.

In the Sinking Gallery, the texts of the final Morse code messages sent and received as the ship was sinking proved powerful in terms of arousing visitors' emotions. The following example is an excerpt of a Morse code message that was sent by the SS Cape Race to the SS Virginia at about 1.40 am, as the Titanic was sinking:

Please tell your captain this: The Olympic is making all speed for Titanic. You are much nearer to Titanic. Titanic is already putting women off in the boats, and he says the weather there is calm and clear. The Olympic is the only ship we have heard says" Going to the assistance of Titanic". The others must be a long way from Titanic (Access at Titanic Belfast, n.d.).

However, the existing AD does not refer to this or any of the Morse code messages presented visually on the wall panels. In fact, no AD at all is provided in the Sinking Gallery: it relies instead on the external audio of real survivors' stories played on a loop. The content selection and decision making on the part of the audio describer, but also the AD users themselves, can significantly affect BPS visitors' emotional experience here. However, because of the looped play of the external audio of the real survivors' stories, visitors usually miss the beginning of the story and finish their visit quickly, thereby truncating the experience. This also explains why a BPS visitor's family member cried and she didn't know why (see Section 1.1). A quotation from another visitor also reflects this doubt: "I think the sinking is a crucial part of the experience. Was there more thay we did not see? It only gives you the survivors' stories. I personally would like more description."

Following on from this, another reason for the diminished emotional experience of BPS visitors is the massive digital culture of museums. With the emphasis on interaction and participation, digital museum culture remains, however, highly reliant on the visual: for example, the darkness in the Sinking Gallery and the silent video showing Titanic sinking at a specific angle. Following the sharing 
of our evaluation report of BPS visitors' experience, Titanic Belfast staff plan to refresh the accessibility offerings in the Sinking Gallery.

\section{Examples of Hedonic Experience}

Museums today, increasingly dependent on recorded footfall, tend to stress the "have fun" kind of experience (Van Aalst \& Boogaarts, 2002). This experience may be felt to encompass enjoyment, excitement, fun, amusement, and indulgence, and was commented upon in the context of different galleries by the participants.

\section{Examples of Introspective Experience}

In the analysis of qualitative data, introspective experience was given substantial expression by BPS visitors. Participants experienced imagining living in other times and places, recalling other experiences, and a sense of belonging and connectedness. Quotes from participants: "The narration of stories gets you more involved. It made you believe that you were actually there"; "I was born and grown in Belfast. On the launch day, it would have been a big day. I can imagine what it was like. The commentary enhanced the whole thing".

\section{Examples of Cognitive Experience}

As a long standing and well-established concept, museums see themselves as playing an educational role. In connection to this, museum expert Hooper-Greenhill (2000) has argued that "the relationship of a museum to its visitors is frequently discussed in terms which prioritise an educational relationship" (p. 17). Cognitive experience is well expressed by the BPS participants in terms of learning something new, historical reminiscence and the experience of novelty.

\section{Examples of Restorative, Transformative and Spiritual Experience}

There were few comments on restorative, transformative and spiritual experience facets, although the overall experience had the potential to be transformative for some visitors. One BPS participant notes, for example: "It opened a whole new world for me. I had never experienced anything like that before". Another said:

It was well thought out. It is a place I have never been to. I am delighted to be able to come. It is a new experience. I was struck by the loss of life and how the families were split up. You can relate to those experiences.

\section{Examples of Inclusive and Empowered Experience}

The 10-facet model covered most of the visitor experiences of the BPS visitors. However, there were two facets reported by the BPS visitors which were difficult to accommodate within the model. These are: inclusive experience, and empowered experience. 
The inclusive experience can be expressed as a sense of being embraced, "being treated fairly and equally" ("Inclusive," n.d.). One of the participants provided feedback on the AD, noting that "I liked the way it told you what was on your right or left. It really told you what a sighted person would see. It is $100 \%$ inclusive". He continued, "It made me feel included".

The empowered experience can be described as "confident and in control of your life, and having the official authority or freedom to do something" ("Empowered," n.d.). One of the participants provided his feedback on his overall experience, and said, "Usually, I am not into museums because of the visual element. It was great to have the $A D$ to describe it for me. It gave me a feeling of control and independence". He also suggested that more navigational information should be included in the AD, and a Pause and Go Back function should be incorporated into the AD device, which would effectively empower him to control his own experience. He notes tellingly, "could you pause the descriptions? This might be useful for directions. Navigation and directions are empowering".

\section{$\underline{\text { Interrelations }}$}

Some of the above themes interrelate. The following are some examples of such interrelations:

(a) The multi-sensory aspects stimulate introspective experience.

E.g., "It was good to feel the heat, the fires; you really picture what it was like at the time."

(b) The multi-sensory aspects stimulate negative emotional response.

E.g., "The heat and the noise made me afraid. It was awful".

(c) The cognitive aspects stimulate introspective experience.

E.g., "The narration of stories gets you more involved. It made you believe that you were actually there."

The interrelations demonstrated above show the complexity of the visitor experience, but these interrelations need more systematic analysis. Furthermore, positive experiences are well categorised in the literature, but how to analyse and categorise negative experiences and the inclusive experience needs further research.

\subsection{Application of DoVE Adjective Checklist}

As there are two new facets in our analysis - the empowered and inclusive experiences-which are not covered by Packer and Ballantyne's 10-facet model of visitor experience, in this section we turn to their later DoVE adjective checklist (Packer, Ballantyne, \& Bond, 2018).

The DoVE adjective checklist has 15 dimensions rather than 10 facets. The "togetherness" dimension is explained as "I felt sociable; I experienced a sense of togetherness, fellowship, companionship, 
community" which we took to cover the inclusive experience we analysed (Packer et al., 2018, p. 221). The "autonomy" dimension is described as "I felt independent, confident; I experienced a sense of choice, control, deciding", which we took to cover the empowered experience we identified (Packer et al., 2018, p. 221). Figure 4 illustrates the mapping of our analysis in Figure 3 (the 10 facet model +2 extra facets) into the 15 dimensions of the DoVE adjective checklist.

Figure 4.

Translation of 10 Facets into 15 Dimensions of the Visitor Experience

\begin{tabular}{ll}
\hline 10 facets & 15 dimensions \\
\hline Physical experiences & Physical activity \\
Hedonic experiences & Excitement \\
Sensory experiences & Aesthetic appreciation \\
Restorative experiences & Peacefulness \\
Relational experiences & Togetherness (inclusiveness) \\
Spiritual experiences & Spiritual engagement \\
Cognitive experiences & Attention \\
& Fascination \\
Emotional experiences & Privilege \\
& Compassion \\
Introspective experiences & Reflective engagement \\
& Connection \\
Transformative experiences & Autonomy (empowerment) \\
- & Personal growth \\
\hline
\end{tabular}

Source: Packer et al., 2018, p. 217.

As mentioned, the 15-dimension checklist is sufficient to embrace the extra accessibility categories identified in our study, whereas the 10-facet model was not sufficiently clear on this issue.

\section{Conclusions}

In this paper, we have evaluated the existing AD and visitor experience at Titanic Belfast with 13 BPS visitors. Having gathered the data, we first applied Packer and Ballantyne's 10-facet model to the visitor experience. To our knowledge, our study is the first application of this model in the context of accessibility for BPS visitors. From the qualitative data analysis based on the reception study, the comments show that the overall experience was very positive. However, the 10-facet model of visitor experience (Packer \& Ballantyne, 2016) was not expressive enough to include accessibility issues. We identified two additional accessibility related facets-Inclusive and Empowered. We then considered the later DoVE adjective checklist of visitor experience by Packer et al. (2018). We noted this checklist is sufficiently expressive to include the accessibility issues. This suggests that when evaluating visitor experience including accessibility, the DoVE checklist may be more appropriate. The study has led to 
some recommendations to further improve the visitor experience and the audio description at Titanic Belfast for BPS visitors. Our subsequent work is focusing on the design of multisensory interactive models which offer a solution as to the amount of $A D$ which can be selected interactively by the user. $A$ voice-driven interactive $A D$ device is also being developed.

In conclusion, museum visitor experience is a complex issue in its own right, but even more complex for BPS visitors. While it is recognised that each visitor is a unique individual and brings different identity-related motivations to museums, this is even more true for BPS visitors, who bring a range of additional-but also variable-factors to bear on the visitor experience. Museum AD is an important access facility for BPS visitors, but it does not in itself guarantee the best visitor experience. This makes evaluating the $A D$ in a museum context more complex in terms of assessing accessibility. It is hoped that the issues identified in this research will alert other researchers to such complexities, and contribute towards increasing the quality of accessibility for BPS museum patrons.

\section{References}

A Titanic number of visitors for top NI tourist spot. (2018, April 16). BBC News. Retrieved from https://www.bbc.co.uk/news

Access at Titanic Belfast. (n.d.). Retrieved from https://titanicbelfast.com/Site/Accessibility.aspx Aho, S. K. (2001). Towards a general theory of touristic experiences: Modelling experience process in tourism. Tourism Review, 56(3/4), 33-37.

Alelis, G., Bobrowicz, A., \& Ang, C. S. (2013, July). Exhibiting emotion: Capturing visitors' emotional responses to museum artefacts [Paper presentation]. In A. Marcus (Eds.), Design, user experience, and usability. The $15^{\text {th }}$ International Conference on Human-Computer Interaction (pp. 429-438). Heidelberg: Springer.

Alexander, E. P., Alexander, M., \& Decker, J. (2017). Museums in motion: An introduction to the history and functions of museums. Lanham: Rowman \& Littlefield.

Bedigan, K. M. (2016). Developing emotions: Perceptions of emotional responses in museum visitors. Mediterranean Archaeology and Archaeometry, 16(5), 87-95. Retrieved from https://doi.org/10.5281/zenodo.204969

Berrios, R. (2019). What is complex/emotional about emotional complexity? Frontiers in Psychology, 10(July), 1606-1616. Retrieved from https://doi.org/10.3389/fpsyg.2019.01606

Cachia, A. (2013). Talking blind: Disability, access, and the discursive turn. Disability Studies Quarterly, 33(3). Retrieved from https://dsq-sds.org/article/view/3758/3281

Caro, M. R. (2016). Testing audio narration: The emotional impact of language in audio description. Perspectives: Studies in Translatology, 24(4), 606-634. Retrieved from https://doi.org/10.1080/0907676X.2015.1120760

Cruz-Garza, J. G., Brantley, J. A., Nakagome, S., Kontson, K., Megjhani, M., Robleto, D., \& ContrerasVidal, J. L. (2017). Deployment of mobile EEG technology in an art museum setting: Evaluation of signal quality and usability. Frontiers in Human Neuroscience, 11, 527-544. Retrieved from https://doi.org/10.3389/fnhum.2017.00527

Csikszentmihalyi, M., \& Robinson, R. E. (1990). The art of seeing: An interpretation of the aesthetic encounter. California: Getty Publications. 
Del Chiappa, G., Andreu, L., \& Gallarza, M. G. (2014). Emotions and visitors' satisfaction at a museum. International Journal of Culture, Tourism, and Hospitality Research, 8(4), 420-431. Retrieved from https://doi.org/10.1108/IJCTHR-03-2014-0024

Dubé, L., \& Le Bel, J. L. (2003). The content and structure of laypeople's concept of pleasure. Cognition and Emotion, 17(2), 263-295. Retrieved from https://doi.org/10.1080/02699930302295

Ekman, P. (1999). Basic emotions. In T. Dalgleish \& M. J. Power (Eds.), Handbook of cognition and emotion (pp. 45-60). Chichester: John Wiley \& Sons.

Empowered. (n.d.). In Cambridge Dictionary online. Retrieved from https://dictionary.cambridge.org/dictionary/english/empowered

Explore. (n.d.). Retrieved from https://titanicbelfast.com/Explore.aspx

Falk, J. H. (2016). Identity and the museum visitor experience. London: Routledge.

Falk, J. H., \& Dierking, L. D. (2000). Learning from museums. Oxford: Rowman \& Littlefield.

Falk, J. H., \& Dierking, L. D. (2016). The museum experience. New York: Routledge.

Falk, J. H., \& Gillespie, K. L. (2009). Investigating the role of emotion in science center visitor learning. Visitor Studies, 12(2), 112-132. Retrieved from https://doi.org/10.1080/10645570903203414

Fryer, L. (2016). An introduction to audio description. London: Routledge. Retrieved from https://doi.org/10.4324/9781315707228

Greco, G. M. (2016). On accessibility as a human right, with an application to media accessibility. In A. Matamala \& P. Orero (Eds.), Researching audio description: New approaches. London:

Palgrave Macmillan (pp. 11-33). Retrieved from https://doi.org/10.1057/978-1-137-56917-2

Hooper-Greenhill, E. (2000). Museum and the interpretation of visual culture. New York: Routledge.

Hutchinson, R. S., \& Eardley, A. F. (2019). Museum audio description: The problem of textual fidelity. Perspectives: Studies in Translation Theory and Practice, 27(1), 42-57. Retrieved from https://doi.org/10.1080/0907676X.2018.1473451

Inclusive. (n.d.). In Cambridge Dictionary online. Retrieved from https://dictionary.cambridge.org/dictionary/english/inclusive

Irwin, H. (2012, April 2). Inside Titanic Belfast-a guided tour. Belfast Telegraph. Retrieved from https://www.belfasttelegraph.co.uk/.

Kim, J. H., \& Ritchie, J. R. B. (2014). Cross-cultural validation of a memorable tourism experience scale (MTES). Journal of Travel Research, 53(3), 323-335. Retrieved from https://doi.org/10.1177/0047287513496468

Liljander, V., \& Strandvik, T. (1997). Emotions in service satisfaction. International Journal of Service Industry Management, 8(2), 148-169. Retrieved from https://doi.org/10.1108/09564239710166272

Matamala, A., \& Orero, P. (Eds.). (2016). Researching audio description: New approaches. London: Palgrave Macmillan

Matamala, A., Soler-Vilageliu, O., Iturregui-Gallardo, G., Jankowska, A., Méndez-Ulrich, J.-L., \& Ratera, A. S. (2020). Electrodermal activity as a measure of emotions in media accessibility research: Methodological considerations. JoSTrans: Journal of Specialised Translation, 33, 129-151.

Neves, J. (2013). Multi-sensory approaches to (audio) describing the visual arts. MonTI. Monografías de Traducción e Interpretación, 4, 277-293. Retrieved from https://doi.org/10.6035/monti.2012.4.12 
Packer, J., \& Ballantyne, R. (2016). Conceptualizing the visitor experience: A review of literature and development of a multifaceted model. Visitor Studies, 19(2), 128-143. Retrieved from https://doi.org/10.1080/10645578.2016.1144023.

Packer, J., Ballantyne, R., \& Bond, N. (2018). Developing an instrument to capture multifaceted visitor experiences: The DoVE adjective checklist. Visitor Studies, 21(2), 211-231. Retrieved from https://doi.org/10.1080/10645578.2018.1553925.

Ramos, M. (2015). The emotional experience of films: Does audio description make a difference? The Translator, 21(1), 68-94. Retrieved from https://doi.org/10.1080/13556509.2014.994853.

Remael, A., Reviers, N., \& Vercauteren, G. (2015). Pictures painted in words: ADLAB audio description guidelines. Trieste: EUT.

Russell, J. A. (1980). A circumplex model of affect. Journal of Personality and Social Psychology, 39(6), 1161-1178. Retrieved from https://doi.org/10.1037/h0077714 .

Snyder, J. (2010). Audio description guidelines and best practices. American Council of the Blind's Audio Description Project. Retrieved from http://www.hestories.info/download/americancouncil-of-the-blinds-audio-description-project-audio.doc .

The shipyard Ride: Take a tour through the shipyard on our electronic dark ride. (n.d.). Retrieved from https://titanicbelfast.com/Explore/TitanicExperience.aspx .

The Titanic Experience. (n.d.). Retrieved from https://titanicbelfast.com/Explore/TitanicExperience.aspx .

Van Aalst, I., \& Boogaarts, I. (2002). From museum to mass entertainment: The evolution of the role of museums in cities. European Urban and Regional Studies, 9(3), 195-209.

Walczak, A., \& Fryer, L. (2017). Creative description: The impact of audio description style on presence in visually impaired audiences. British Journal of Visual Impairment, 35(1), 6-17. Retrieved from https://doi.org/10.1177/0264619616661603.

World's Leading Tourist Attraction 2016. (2016). Retrieved from https://www.worldtravelawards.com/award-worlds-leading-tourist-attraction-2016. 